\title{
Modelling the Contact between Wheel and Rail within Multibody System Simulation
}

\author{
GUNTER SCHUPP $^{1}$, CHRISTOPH WEIDEMANN $^{2}$ AND LUTZ MAUER ${ }^{2}$
}

\begin{abstract}
SUMMARY
In modern railway industry the simulation of the behaviour of railway vehicles has become an important design method during the last years. Modern simulation packages offer modelling elements that are highly adapted for standard and unusual simulation scenarios. A specific application case is the simulation of a railway vehicle travelling through a switch. It makes high demands on the simulation software due to the inconvenient modelling elements needed: the changing rail profiles on the blade rail and in the crossing vee area as well as the guard rail with its additional contact at the back of the wheel. The article gives an overview over the state of the art in railway vehicle simulation and presents a simulation of a passenger car running through a switch as an application example.
\end{abstract}

Keywords: Multibody system simulation, railway vehicle dynamics, wheel rail contact, nonconstant rail profile cross-sections, running trough a switch.

\section{INTRODUCTION}

An important range of application for computer aided multibody system simulations is the analysis and the design of a railway vehicle's running behaviour. For general vehicle systems, as well as for general mechanical applications, such software simulations represent an efficient way to accomplish the growing need for cost and time reduced development cycles of new vehicles. Wheel-rail systems, and therefore also their simulations, can be characterized by two outstanding properties: the vehicle is guided along an arbitrary track and the contact between steel wheel and steel rail means large contact forces transmitted via small contact areas. Hence, a simulation software for the analysis of general mechanical systems must be enhanced by adequate and highly specific modelling features before it can be applied to railway vehicles.

The article concentrates on those railway specific peculiarities. The first, rather short, part is about modelling arbitrary railway vehicles as multibody systems within

${ }^{1}$ Address correspondance to: DLR - German Aerospace Center, Institute of Aeroelasticity, Postfach 1116, 82230 Wessling, Germany. Tel.: +49-8153-28-2412. E-mail: Gunter.Schupp@dlr.de

${ }^{2}$ INTEC GmbH, Argelsrieder Feld 13, 82234 Wessling, Germany. 
a generally applicable simulation environment. The concurrent application of other appropriate engineering software tools like CAD or FEA facilitates or enables then the design of unconventional vehicle concepts. The main focus is built by the second part where an efficient modelling of the highly complex interaction between the wheels of a vehicle and the rails is illustrated, implemented within the wheel-rail module of the simulation software SIMPACK. The section closes with the description of a virtual representation of switches (points) characterized by rail cross-sections varying along the track. This specific feature is an essential prerequisite for the realistic simulation of railway vehicles running through a switch, described within the application part.

\section{ON THE MODELLING OF RAILWAY VEHICLES}

The first step of every vehicle simulation on the computer is to set up a mechanical model appropriate to fulfil the desired simulation task. This model constitutes the basis for the mathematical description, the equations of motion, obtained with the aid of physical principles and laws (Newton's laws etc.). The multibody system (MBS) approach is a powerful and widely used method for this procedure, especially if a vehicle's running behaviour is to be analyzed/designed. To avoid the time-consuming and error-prone task of compiling the mathematical model as system of equations by hand, suitable professional software packages built upon this approach are commercially available. They provide the engineer not only with software tools for the model set up but usually allow also the application of a wide range of different numerical algorithms on the automatically generated system equations in a way optimized for the specific modelling and the simulation task. A comprehensive survey of such simulation software in the sphere of wheel-rail systems is given in [1]. The following descriptions are based on the simulation package SIMPACK, [2], a general multibody simulation tool with an extensive wheel-rail functionality; for a detailed monograph about the modelling and simulation of (railway) vehicles see e.g. [3].

For analysing the dynamic behaviour of railway vehicles running on arbitrary tracks under arbitrary manoeuvres, usually the vehicle (and the maybe necessary environment) is abstracted basically as a multibody system, see fig. 1. A multibody system consists of rigid or elastic bodies, interconnected via massless force elements and joints. Due to the relative motion of the system's bodies, force elements generate applied forces and torques. Typical examples are springs, dampers and actuators combined to primary and secondary suspensions of railway vehicles. Contrarily, joints give rise to constraint forces by constraining the relative motion of the system's bodies. The scope of applications starts with simple single axis rotational joints and ends with highly complicated and specific ones like the so-called 'wheel-rail joints' guiding bodies along arbitrary tracks. Usually, the user can rely on extensive libraries of connecting elements while setting up the simulation model. 

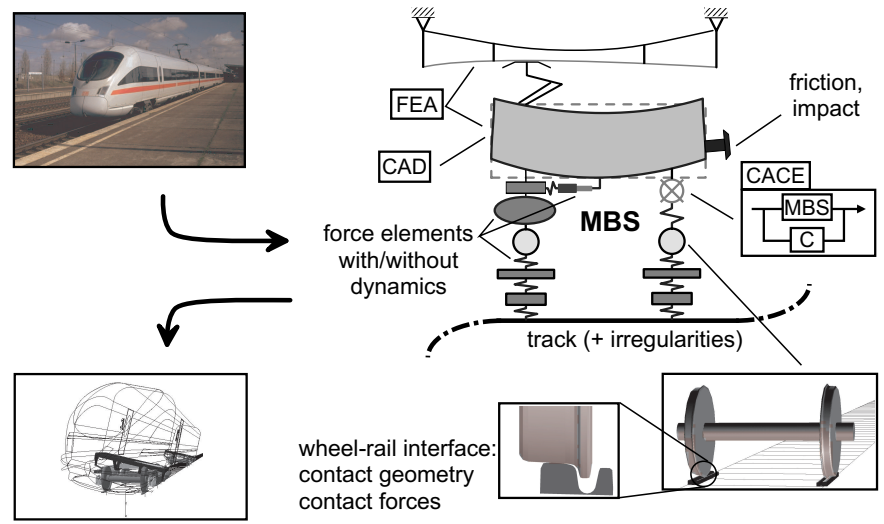

Figure 1. Generic simulation model for design and analysis of a railway vehicle's running behaviour. (Photograph: C. Splittberger, Internet: http://mercurio.iet.unipi.it.)

To serve the purpose of modern and future design criteria, bi-directional interfaces to well-known and established Computer Aided Engineering (CAE) software are required additionally to reproduce specific phenomena. Thus, certain simulation tasks and requirements are easier to be performed - or become accomplishable at all, [4].

To take the flexibility of lightweight structures into account, an interface to different Finite Element (FEA) software has been implemented. Here, in a first step, a number of mode shapes of the body to be modelled elastically are calculated by FEanalysis. The results are then transferred to the multibody simulation, resulting in a 'hybrid' model consisting of rigid and elastic bodies. Finally, on behalf of a dynamic stress analysis of the flexible body's most endangered locations, the dynamic forces and accelerations following from the time-integration of the system equations can be shifted back to the FE software, see also [5].

More and more electronically controlled elements like tilting systems etc. are implemented in vehicles to shorten overall travel times, to increase ride comfort and so on. One possibility to incorporate active/semi-active (i.e. feed back control) elements into the simulation models of such mechatronic systems is to use internal controller design tools. For more advanced or more complex controller design tasks, a bidirectional interface to Computer Aided Control Engineering (CACE) software such as MATLABSIMULINK can be applied.

To incorporate physical and graphical Computer Aided Design (CAD) data, thus extending the graphical abilities of the software and concurrently making MBS setup faster, easier and more secure, an interface to CAD software exists. Vice versa SIMPACK can be used to support the design process within CAD software itself by dynamic, quasi-static and kinematic analyses. 
A characteristic feature of railway vehicles is the guidance of the vehicle along a track. To simulate manoeuvres on arbitrary tracks, standard track models like curve entries, cross overs, etc. might be defined or measured tracks can be incorporated directly into the model. In reality, the layout of a track is superimposed with (usually) small irregularities. For realistic simulations, those irregularities can be defined either as lateral, vertical and roll (about longitudinal axis) deviations from the ideal track centre line, possibly with a gauge irregularity superimposed, or for right and left rail separately. Stochastic irregularities can be defined either directly as measured data or by the power spectral density, i.e. as a stochastic, stationary process, [3].

But the distinctive feature of railway vehicles and their simulation is the contact between steel wheel and steel rail with the profile cross sections of wheel and rail as crucial parameters. This interface is described next.

\section{MATHEMATICAL DESCRIPTION OF THE WHEEL-RAIL INTERFACE}

An important prerequisite for the simulation of railway vehicles equipped with the rather traditional rigid wheelset as well as of vehicles equipped with independently suspended wheels is the availability of a general wheel-rail contact module describing fundamentally the contact between one wheel and the rail. This is also the foundation of SIMPACK's wheel-rail functionality which will be pointed out in the sequel; a more detailed description is given in [2] and [6].

\subsection{Equations of motion}

Starting from an interpretation of the contact between wheel and rail as a kinematically closed loop which is described as kinematic constraint, the equations of motion of a vehicle can be formulated in the position coordinates $\mathbf{p}(t)$ and the velocity coordinates $\mathbf{v}(t)$ as Lagrange equations of the first kind,

$$
\begin{aligned}
\dot{\mathbf{p}} & =\mathbf{v} \\
\mathbf{M}(\mathbf{p}, t) \dot{\mathbf{v}} & =\mathbf{f}(\mathbf{p}, \mathbf{v}, \boldsymbol{\lambda}, t)-\mathbf{G}^{T}(\mathbf{p}, t) \boldsymbol{\lambda} \\
\mathbf{0} & =\mathbf{g}(\mathbf{p}, t),
\end{aligned}
$$

a system of differential-algebraic equations (DAE). With the contact condition between wheel and rail formulated in a suitable manner as algebraic constraint (3), the Lagrangian multipliers $\boldsymbol{\lambda}(t)$ correspond to the normal forces acting in the points of contact normally to the profiles of wheel and rail $(\mathbf{G}(\mathbf{p}):=\partial \mathbf{g}(\mathbf{p}) / \partial \mathbf{p})$. Thus, the vector $\mathbf{f}(\mathbf{p}, \mathbf{v}, \boldsymbol{\lambda}, t)$ of the applied forces (which includes also the Coriolis forces, etc.) depends directly via the friction forces between wheel and rail on the multipliers $\boldsymbol{\lambda}(t)$. The symmetric mass matrix is denoted $\mathbf{M}(\mathbf{p})$. 
Replacing the constraint equations (3) by a stiff 'Hertzian contact spring', i.e. by a one-sided spring-damper force element, the equations of motion yield as a system of ordinary differential equations (ODE), $\dot{\mathbf{y}}=\mathbf{f}_{e}(\mathbf{y}, t), \mathbf{y}:=\left(\mathbf{p}^{T}, \mathbf{v}^{T}\right)^{T}$. A typical application of this 'elastic' contact model would be the investigation of wheel-lift scenarios or derailments, but, particularly under a numerical point of view, the preceding 'constrained contact' model ought to be preferred.

\subsection{Contact geometry: A quasi-elastic contact model}

Let $d(s, \mathbf{q})$ be the distance between two corresponding points on the wheel and the rail envelope curves in vertical direction being parameterized by the lateral wheel profile coordinate $s$ and the relative position vector $\mathbf{q}$ of the wheel with respect to the rail. Since the position vector $\mathbf{q}:=\mathbf{q}(\mathbf{p}, t)$ depends on the MBS coordinates $\mathbf{p}$ of eqs. (1)(3), the classical contact condition

$$
g(\mathbf{q}):=\min _{s} d(s, \mathbf{q})=0
$$

also defines directly the kinematic constraint (3). The lateral coordinate $\bar{s}$ of the contact point on the wheel follows then from the necessary condition

$$
\left.\frac{\partial}{\partial s} d(s, \mathbf{q})\right|_{s=\bar{s}}=0 .
$$

The crucial point of this 'rigid contact' model is the differentiability of equation (4). For so-called wear profiles - in fig. 2 (left) the combination S1002/UIC60-ORE is given exemplarily - the location of the contact point $\bar{s}:=\bar{s}(\mathbf{q})$ varies discontinuously with respect to $\mathbf{q}$, see fig. 2 (right). To guarantee the required (and more realistic!) smoothness of the equations of motion, the basic principle described in [6], and especially also in [7], is to take into account qualitatively the elastic deformation of the wheel-rail interface on the whole contact patch. Within the resulting 'quasi-elastic contact' model the contact condition (4) is replaced by

$$
g(\mathbf{q})=\varepsilon \ln \left(\int_{s_{\min }}^{s_{\max }} \exp \left(\frac{1}{\varepsilon} d(s, \mathbf{q})\right) d s / \int_{s_{\min }}^{s_{\max }} d s\right)=0
$$

with $\varepsilon$ being a small positive parameter. Introducing the weighting function $w(s, \mathbf{q}):=$ $\exp (d(s, \mathbf{q}) / \varepsilon)$ then leads to the weighted mean value of the profile coordinate $s \in$ $\left[s_{\min } ; s_{\max }\right]$ as the lateral contact point location $\bar{s}$,

$$
\bar{s}=\int_{s_{\min }}^{s_{\max }} s \cdot w(s, \mathbf{q}) d s / \int_{s_{\min }}^{s_{\max }} w(s, \mathbf{q}) d s .
$$


The success of this algorithm compared to the rigid contact model is demonstrated by fig. 2, right.
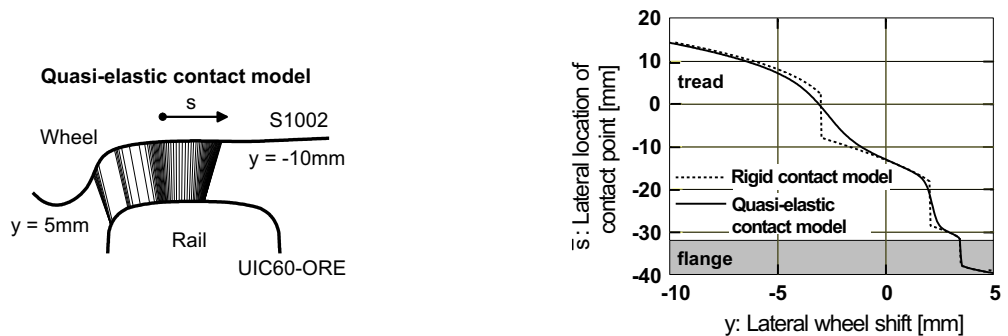

Figure 2. Left: Profile cross-sections of wheel (S1002) and rail (UIC60-ORE) including the potential points of contact. Right: Lateral contact point location $\bar{s}$ on the wheel with the relative lateral shift $y$ between wheel and rail varied.

A considerable speed up of the simulations can be gained by a two-dimensional spline approximation of the contact condition (6), the contact point location (7) and further frictional parameters in combination with their symmetric quadratic interpolation. The approximation is performed in terms of the lateral shift and the roll angle of the wheel relative to the rail, the interpolation has the according yaw angle as independent variable. For that purpose, the necessary spline coefficients are computed and stored as table on file during model setup in a pre-processing step. Then, during simulations only these tables have to be evaluated.

\subsection{Contact mechanics: handling the contact forces}

The contact forces acting in the point of contact are subdivided into normal forces and tangential friction (creep) forces. As already described in section 3.1, the normal forces coincide with the Lagrangian multipliers $\lambda$ in the DAE-case; in the ODE-case the normal forces follow as applied forces from a spring-damper characteristic.

The default assumption of just one point of contact per wheel can be replaced by a multiple contact model, allowing up to three different points of contact per wheel: one on the tread, one on the flange and a third one again on the flange or on the back of the wheel. Typical applications are simulations comprising e.g. channel rails, heavily worn profiles of wheels and rails and curved tracks with small curve radii. Another field would be the simulation of vehicles running through a switch. The successful and efficient application of any multi point contact model demands an algorithm that guarantees a continuous distribution of the constraint force $\lambda$ among the points of contact with respect to relative kinematics. And, second, the vector sum of the normal forces (per wheel) has to be mechanically equivalent to the constraint force.

The calculation of the tangential creep forces is based on Hertzian (elliptical) contact properties and usually performed by the code FASTSIM which applies Kalker's 
simplified theory of rolling contact, [8]. Other friction laws, some of them with the advantage of being analytically describable, are also in use, see e.g. [9]. Compared to a computationally more expensive non-Hertzian contact description, the Hertzian contact model has the advantage that it can be characterized by four parameters (ratio of the semi axes of an equivalent contact ellipse and three normalized creepages). Therefore, using the quasi-elastic contact model, the main curvatures of wheel and rail defining the geometrical properties of the contact ellipse are obtained also by a weighted sum approach, analogously to the calculation of the contact point location with eq. (7).

\subsection{Non-constant rail profiles}

For the simulation of switch crossings the usual assumption of left and right rail showing each a constant profile cross-section is no longer sufficient to reproduce relative motions and particularly the interacting forces in the wheel-rail interface satisfactorily. Therefore, for such simulations the profiles of the rails' cross-sections have to vary along the track, i.e. so-called 'non-constant rail profiles' become necessary. Some characteristic rail cross-sections around the crossing vee are given exemplarily in fig. 3, right. Though the simulation of switch crossings is the most important simulation task requiring varying rail cross-sections, it is not the only one.
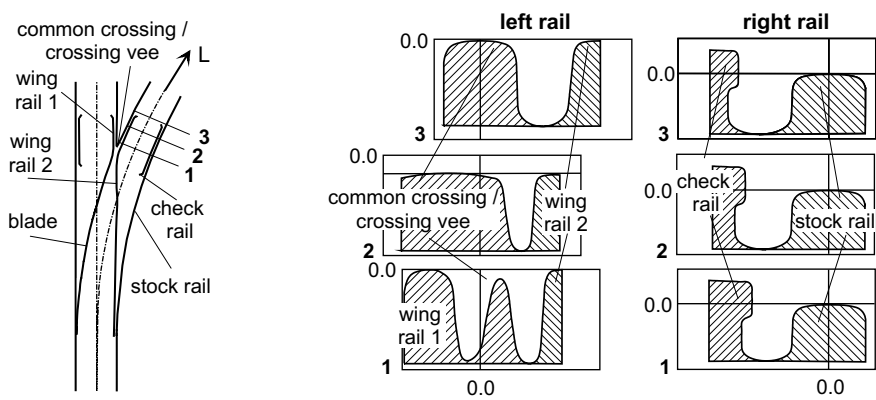

Figure 3. Left: Schematic top view of a switch (points) with some characteristic denominations. Right: Selected rail profile cross sections around the common crossing.

A switch (points) can be subdivided into a couple of different single rails like the wing and the check rails etc., see fig. 3. Within its virtual representation these single rails are merged into altogether four different assemblies of rail cross-sections. These assemblies represent the 'blade - wing rail 1 - common crossing' for contact with the tread/flange of left wheels, the 'stock rail' for contact with the tread/flange of right wheels, the 'wing rail 2' for contact with the back of left wheels and the 'check rail' for contact with the back of right wheels. The given connection between rail fragment 
and wheel part is valid only for the crossing scenario shown in fig. 3, left, i.e. if the vehicle follows the route turning out on the right hand side of the straight track.

A rail assembly is defined via the measured rail profiles at relevant cross-sections, [6], marked with ' $X$ ' in fig. 4; this figure also indicates the complete approximation procedure. For its application in subsequent simulations, the complete measurement data defining a switch is prepared just once per switch in three sequential, preparatory steps: In the first step, a smoothing cubic spline representation is generated for each measured cross-section $i$. With $\mathbf{v}_{i}, \mathbf{g}_{i}$ collecting the lateral and the vertical profile coordinates of cross-section $i$, respectively, and the normalized arc-length parameter $u$, each of these representations is based on a normalized parametric formulation of the rail profile function $\mathbf{g}_{i}=\mathbf{g}_{i}\left(\mathbf{v}_{i}\right)$,

$$
\left.\begin{array}{l}
\mathbf{v}_{i}=\mathbf{v}_{i}(u) \\
\mathbf{g}_{i}=\mathbf{g}_{i}(u)
\end{array}\right\} u \in[0 ; 1] .
$$

In view of the finally necessary interpolation between the cross-sections, the next step is to generate a pointwise version of the profile function discretized equidistantly with respect to the curve parameter $u$ (dots ' $\bullet$ ' within fig. 4). Here it is important to use one and the same $u$-discretization for every cross-section of an assembly. The final preparatory step is then to calculate a once again cubic spline representation of the resulting profile definition and store it on file. During simulation, according to the current location of a wheel with respect to the rail, first a spline representation of an intermediate profile cross-section (grey in fig. 4) is generated by a linear interpolation between the two limiting cross-sections. A final spline approximation then yields the coordinates $v(u), g(u)$ of the profile function, ' $\triangle$ ', for the given curve parameter $u$.

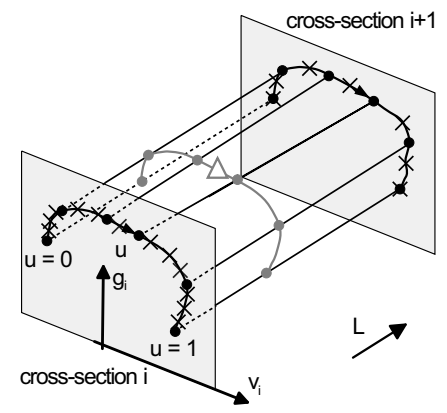

Figure 4. Recovering a rail assembly from measured profile cross-sections by a combined, sequential approximation scheme.

The advantages of this procedure are that arbitrarily and differently discretized measurement data as well as possible undercuts of the profile functions can be handled. But due to the now non-constant rail profiles the highly efficient table evalua- 
tions of the contact geometry and parameters described at the end of section 3.2 have to be replaced by the online mode of their calculation.

\section{APPLICATION EXAMPLE: A RAILWAY VEHICLE RUNNING THROUGH A SWITCH}

\subsection{Areas of Application}

The simulation of a railway vehicle travelling through a switch is to some degree a sophisticated task, as can be seen from the above explanations. In contrast, there are many potential cases of application that are worth examining. In general, the forces acting between wheel and rail are considerably higher in switches and crossings than on standard track. Because of their high dynamics resulting from the rapidly changing rail profile forms they may have a worse influence on the passenger ride comfort and the lifetime of bogie components than narrow curves or track irregularities. For all that, up to now only few realistic data are available about the wheel/rail forces acting within switches and crossings. Hence, such simulations are useful e.g.

- for determining the wheel/rail forces (lateral, $Y$, and vertical, $Q$ ) and their high dynamics within the switch,

- for the generation of load collectives for a series of switches and/or crossings and the corresponding vehicles in a railway system or

- for minimising the load on wheels and rails as well as improving the passenger ride comfort by optimising the profile geometry of the switch.

Another promising application of switch simulation can be the examination of the guidance behaviour or the space that the wheels will require in the switch. Switches are known to have some crucial points that are susceptible to derailments or intense wear: the beginning of the blade rail, especially in the divergent route, or the area near the crossing vee, where the wheelset will be guided by the check rail. In these cases only the simulation is able to predict reliably the position of the wheels in the gauge channel and their addiction to derailment.

\subsection{Unusual modelling features}

Even if 'non-constant rail profiles' as described in section 3.4 are the most important modelling feature for simulations of railway vehicles running through a switch, a couple of extraordinary modelling elements have to be provided additionally to reproduce the unusual contact situations between wheels and rails that have to be anticipated in this case, [6]. Those situations occur e.g. if one wheel is in the vicinity of the crossing vee, see fig. 3; the following descriptions refer to the crossing scenario given there. 
The wheel running over the crossing vee has to change its contact from the blade rail to the through rail (or vice versa), which leads usually to more than one point of contact per wheel - at least one on its tread and one on its flange has to be expected. How this multiple contact situation can be handled has been outlined in section 3.3.

Also around the crossing vee, a wheel can loose its contact with the rail. This wheel lift, indicated by a vanishing constraint force $\lambda=0$, means a structural change of the equations of motion because the constraint or contact condition (6) is no longer valid. Once wheel lift is detected during simulation by means of a root function technique, the constraint between wheel and rail is replaced automatically by the 'elastic' contact model utilizing a one-sided spring-damper element as described in section 3.1.

As long as the wheel runs in the vicinity of the crossing vee, the wheelset is guided by the contact of the opposite wheel's back with the check rail, while the back of the wheel on the crossing vee may contact wing rail 2. Hence, an additional contact element between the back of each wheel and the check/wing rails is necessary. Since those contacts are usually rather short-time, again the 'elastic' contact formulation is chosen for this case.

\subsection{The simulation model}

The vehicle used for the examination is a standard passenger car running on Vignol rail profiles, modelled with the multibody simulation package SIMPACK based on the data of the 'Manchester Benchmark' $[1,10]$. Its overall data can be found in table 1. The model uses standard elements for primary and secondary suspension, that are equipped with linear spring and damper characteristics. For the wheel/rail contact SIMPACK's multi contact model is chosen in order to reproduce the special contact situations at the beginning of the blade rail and on the crossing vee. With this method, up to three different contact points on each wheel are possible. Furthermore, for the guard rail contact also the back-of-wheel contact is active.

A standard switch of type 'EW 60-300-1:9' is used for the examination, which can be found e. g. on feeder lines or in stations in Germany. Its overall data are shown in table 1 as well.

The profile cross sections needed for the modelling of the non-constant rail profiles are taken from a three-dimensional CAD model set up previously according to the technical drawings of the switch [11]. This is necessary because the technical drawings themselves do not depict all the cross sections needed in the simulation. On the contrary, some parts of the changing rail profiles are only given by an explanation of the manufacturing process, e. g. by depicting the tool and its movement when planing the raw rail. Instead of converting the profile coordinates from a CAD model, also measured profile data could be used.

The switch model consists of 55 different cross sections that are used in total 83times at the appropriate positions in the switch. The high number of cross sections is 
Table 1. Overall data of the passenger vehicle and the switch.

\begin{tabular}{ll}
\hline Total mass & $45800 \mathrm{~kg}$ \\
Total length & $26.4 \mathrm{~m}$ \\
Bogie distance & $19 \mathrm{~m}$ \\
Wheelset distance & $2.56 \mathrm{~m}$ \\
Nr. of wheelsets & 4, in 2 bogies \\
Track gauge & $1435 \mathrm{~mm}$ \\
Wheel profile & $\mathrm{S} 1002$ \\
\hline Switch denotation & EW $60-300-1: 9$ \\
Stock rail profile & UIC 60, not canted \\
Travelled route & divergent route, left-hand \\
Switch angle & $6.37^{\circ}(1: 9)$ \\
Radius of divergent route & $300 \mathrm{~m}$, no curve entry/exit \\
Guard rail denotation & Rl 1-60 \\
\hline
\end{tabular}

necessary in order to ensure a correct profile interpolation even between very unalike profile forms. Figure 6 (right) shows the cross-sections used for the blade rail.

Because of the high forces in the crucial regions of the switch and their high dynamics it is necessary to work with an elastic track model. Here a standard track representation is used, consisting of one equivalent mass per wheelset that represents the ballast and the sleeper. It is suspended with respect to the inertia system by means of spring/damper elements. The mass and the (here constant) spring and damper coefficients are taken from [12].

The graphical representation of the switch within SIMPACK is created automatically according to the profile data. It is shown in fig. 5. Figure 6 (left) depicts the crossing vee region in detail.

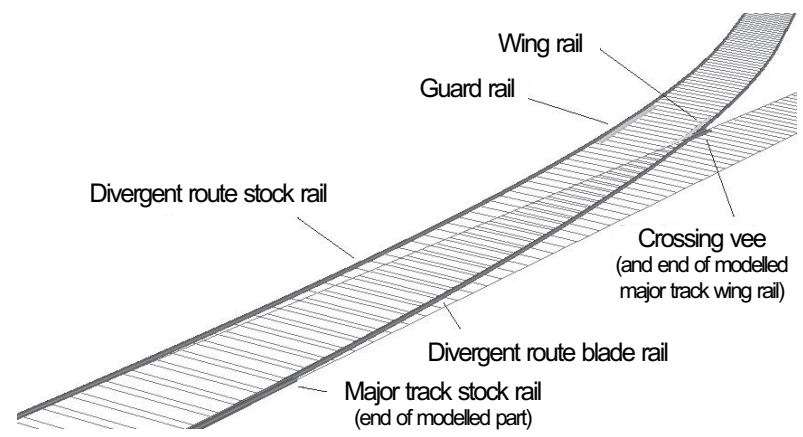

Figure 5. Overview of the switch EW 60-300-1:9 used in the calculation.

In the simulation the vehicle travels on the divergent route with a velocity of $20 \mathrm{~km} / \mathrm{h}$. The total simulation time needed by the vehicle to pass the initial straight 

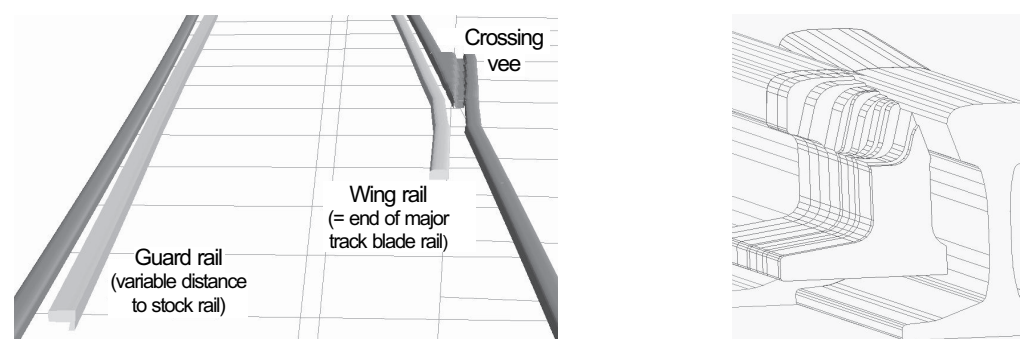

Figure 6. Left: Detail of the crossing vee area in SIMPACK. Right: Cross-sections of the blade rail in the CAD model (contracted).

track and the switch is $15 \mathrm{~s}$. On a $1.7 \mathrm{GHz}$ Pentium IV notebook computer the simulation takes about ten minutes.

\subsection{Results}

The simulation yields a lot of data that give in total a good overview on what happens in the different parts of the switch. Besides the wheel/rail forces one will be interested also e.g. in the position of a wheel / a wheelset in the track. Useful output data of the simulation are also:

- the lateral force $Y$ for a wheel(set) or even for one of the up to three contact points,

- the ratio $Y / Q$ as an indicator for the risk of derailment,

- the normal force $N$ for each of the contact points,

- the lateral force on the back of the wheel touching the guard rail,

- the lateral position $y$ and yaw angle $\psi$ of the wheelset in the track,

- the position of the contact points on wheels, rails and guard rail and

- the dissipated frictional power at the contact patches indicating the material wear.

In fig. 7 the unfiltered wheel/rail forces $Y$ and $Q$ for the leading left and right wheel are shown. The blade rail starts at $50 \mathrm{~m}$, followed immediately by the curve entry with its high dynamics. After a quite stable run in the blade rail, between $78 \mathrm{~m}$ and $80 \mathrm{~m}$ the crossing vee produces again high dynamics. The right wheel shows $Y$ forces on a level of $40 \mathrm{kN}$. The left wheel's $Y$ forces stay generally at a level of about $20 \mathrm{kN}$, but at the travelled distance of $78 \mathrm{~m}$ to $80 \mathrm{~m}$ the wheel experiences a maximum $Y$ force of $36 \mathrm{kN}$.

Figure 8 shows the corresponding derailment coefficients $Y / Q$. The derailment coefficient of the right wheel (which is the outer wheel in the curve) becomes no higher than 0.5 , except of some peaks at a travelled distance of $56 \mathrm{~m}$ and $81 \mathrm{~m}$, that would disappear after applying a $2 \mathrm{~m}$ sliding mean filter. For the left (inner) wheel, 
the peak value of 0.85 appears at the contact of the back of the wheel with the guard rail and is a result of the nearly vertical contact patch orientation in this case.

Running with a speed of $50 \mathrm{~km} / \mathrm{h}$ instead of $20 \mathrm{~km} / \mathrm{h}$ through the switch yields some higher values at the peaks, but after a $2 \mathrm{~m}$ sliding mean filtering the overall derailment coefficients turn out to be not significantly higher. Please note that the simulation has been carried out with a track model of rigidly mounted rails on a flexibly bedded ballast mass. The $Y$ forces would be lower if both rails were mounted separately and flexibly on the ballast mass.

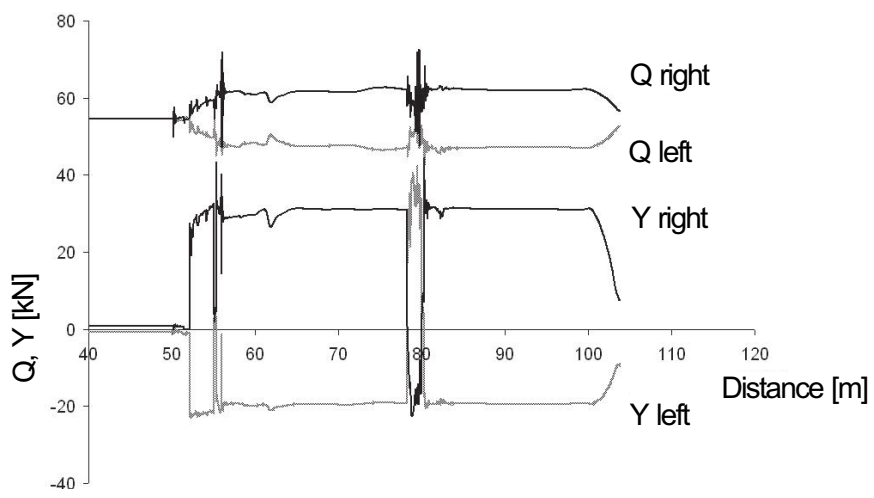

Figure 7. Simulation results: wheel/rail forces $Y$ and $Q$ for the leading wheelset (unfiltered).

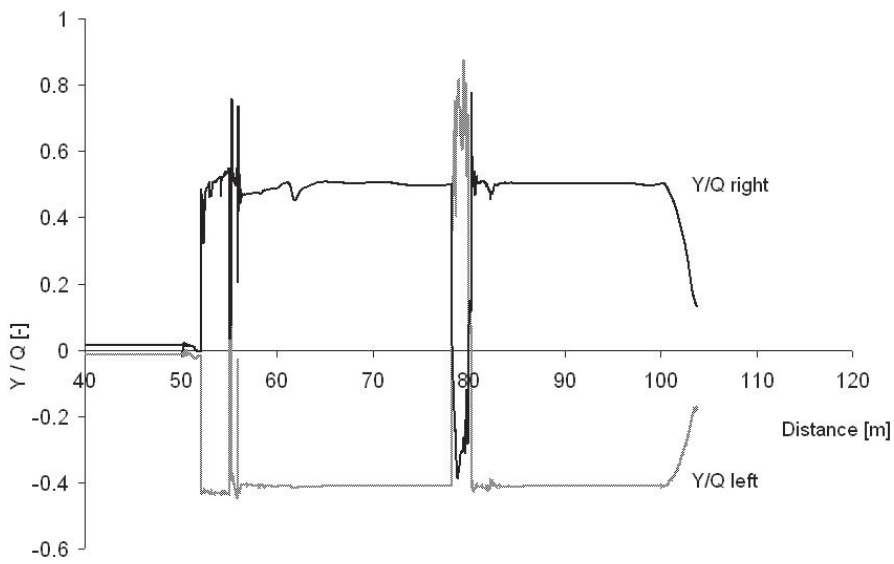

Figure 8. Simulation results: derailment coefficients $Y / Q$ for the leading wheelset (unfiltered). 
Fig. 9 depicts the lateral positions of the up to three contact patches on the leading right wheel at the beginning of the blade rail. Fig. 10 shows four characteristic contact situations at the beginning of the blade rail, they correspond with the according marks in fig. 9. Situation A is $1.45 \mathrm{~m}$ behind the beginning of the blade rail, with still one contact point on the stock rail, even if the blade rail is already noticeable. In situation $\mathrm{B}$ the wheel comes into contact with the stock rail on the one hand and with the blade rail on the other hand, so two contact patches exist. Situation $C$ is the short moment where the wheel has three contacts at the same time. In situation D, now completely on the blade rail, we find a normal curving situation with a contact patch near the flange. It is obvious that the situations B and C are not favourable because of the higher wear resulting from the different rolling radii at the contact patches.

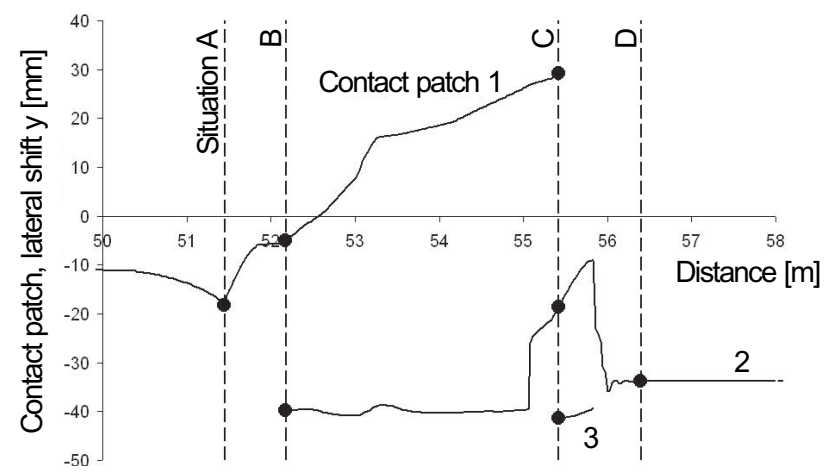

Figure 9. Contact patch lateral positions along the wheel profile at the beginning of the blade rail (leading right wheel), measured with respect to the wheel taper line; negative values towards the flange.

A

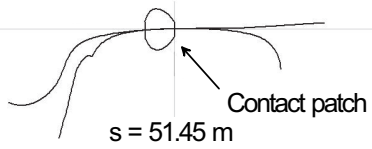

C

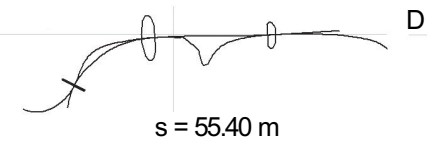

B

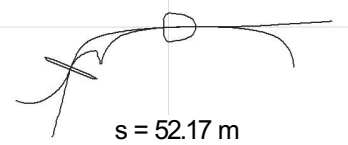

D

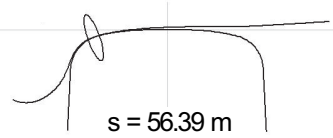

Figure 10. Four different cross sections and contact situations in the blade rail ( $\mathrm{A}-\mathrm{D}$ matches the 'situation $A^{\prime}$ etc. in fig. $9, s$ is the travelled distance). 


\section{CONCLUSIONS}

In the last years, multibody simulation has become a standard tool for the design process in railway industry. Modern simulation packages like SIMPACK offer a wide range of modelling possibilities not only for standard calculations but also for unusual simulation tasks like running through a switch. Calculation times are sufficiently short in order to allow complex examinations where e.g. profile forms have to be optimised.

The described example can be taken as basis for further advanced examinations. One could think e.g. about introducing a track foundation with variable parameters representing the different stiffnesses of the switch parts in a better way. For the study of derailment situations also the guard rail could be mounted elastically in the track.

These extensions will require a better understanding of the elasticities of the different parts of a switch. This can be achieved only by bringing together the simulation with measurements in the reality, and by taking advantage of the big knowledge accumulated by experts in the field. In this respect, the simulation may be regarded as the key for connecting experience and theory.

\section{REFERENCES}

1. S. Iwnicki, editor. The Manchester Benchmarks for Rail Vehicle Simulation, volume 31 of Supplement to Vehicle System Dynamics. Swets \& Zeitlinger, Lisse, 1999.

2. W. Rulka. Effiziente Simulation der Dynamik mechatronischer Systeme für industrielle Anwendungen. $\mathrm{PhD}$ thesis, Technical University of Vienna, Austria, 1998.

3. W. Kortüm and P. Lugner. Systemdynamik und Regelung von Fahrzeugen. Springer, Berlin etc., 1994.

4. G. Schupp and A. Jaschinski. Virtual prototyping: the future way of designing railway vehicles. Int. J. of Vehicle Design, 22(1/2):93-115, 1999.

5. S. Dietz. Vibration and Fatigue Analysis of Vehicle Systems Using Component Modes. FortschrittBerichte VDI Reihe 12, Nr. 401. VDI Verlag, Düsseldorf, 1999.

6. H. Netter. Rad-Schiene-Systeme in differential-algebraischer Darstellung. Fortschritt-Berichte VDI Reihe 12, Nr. 352. VDI Verlag, Düsseldorf, 1998.

7. K. Frischmuth, M. Arnold, M. Hänler, and H. Netter. Differentialgleichungen und singuläre Mannigfaltigkeiten in der dynamischen Simulation von Rad-Schiene-Systemen. In K.-H. Hoffmann, W. Jäger, Th. Lohmann, and H Schunck, editors, Mathematik - Schlüsseltechnologie für die Zukunft, pages 331342. Springer-Verlag, Berlin Heidelberg New York, 1997.

8. J. J. Kalker. Three-Dimensional Elastic Bodies in Rolling Contact. Kluwer Academic Publishers, Dordrecht, Boston, London, 1990.

9. O. Polách. A Fast Wheel-Rail Forces Calculation Computer Code. In The Dynamics of Vehicles on Roads and Tracks, $16^{\text {th }}$ IAVSD-Symposium, Pretoria, South Africa, 1999, pages 728-739. Swets \& Zeitlinger B.V., Amsterdam and Lisse, 2000.

10. S. Iwnicki. Manchester benchmarks for rail vehicle simulation. Vehicle System Dynamics, 30:295-313, 1998.

11. H. Seyedin. Mehrkörperdynamische Simulation von Weichendurchfahrten. Master's thesis, Munich Univ. of Appl. Sc., Inst. Aeronautical Engineering, Germany, 2003.

12. Specification for a bogie with improved curving dynamics. In ERRI B 176 Bogies with steered or steering wheelsets: RP1, Vol. 2. ERRI - European Rail Research Institute, Utrecht, Holland, 1989. 